\title{
Review of Early Intervention for Children with Autism Spectrum Disorder: Focused on Randomized Controlled Trials
}

\author{
Young-Hui Yang \\ Department of Child and Adolescent Psychiatry, National Center for Mental Health, Seoul, Korea
}

\begin{abstract}
Early identification and intervention for autism spectrum disorder (ASD) were reported to be important for outcomes or clinical courses. However, there have been a few robust evidences for effectiveness of early intervention until now. This review aims to identify the effectiveness of early intervention by investigating the randomized controlled trial (RCT) of early intervention for autism. There are some RCT studies using behavioral program. Although there are some significant findings, the outcome measurements and small sample size are the limitations. Further studies are needed.
\end{abstract}

Key Words: Autism spectrum disorder; Early intervention; Randomized controlled trial.

Received: November 27, 2018 / Revision: May 7, 2019 / Accepted: August 28, 2019

Address for correspondence: Young-Hui Yang, Department of Child and Adolescent Psychiatry, National Center for Mental Health, 127 Yongmasan-ro, Gwangjin-gu, Seoul 04933, Korea

Tel: +82-2-2204-0118, Fax: +82-2-2204-0394, E-mail: yhyang12@korea.kr

\section{INTRODUCTION}

Developmental disorders are characterized by delays, deviations, or impairments of functions such as cognition, social interaction, and language in early life. Developmental disorders can be broadly divided into intellectual disorders, autism spectrum disorders (ASD), and language disorders. Intellectual disorders and language disorders are diagnosed in cases where intelligence, adaptive ability, or language development are at least 2 standard deviations below the mean for the patient's age group, and the patient shows a level of dysfunction that restricts daily living. ASD is diagnosed in cases showing persistent deficits or deviations in social interactions and repetitive, restricted patterns of behaviors or interests [1]. These disorders can exist independently but often present together. ASD especially is a complex disorder that can show impairments in intellect, language development, and social interaction simultaneously.

Intellectual disorders typically persist throughout a person's entire lifetime, but the course of the disease can differ depending on intelligence quotient, environmental support, and the underlying medical or genetic conditions. Daily function can be expected to improve with special education and

This is an Open Access article distributed under the terms of the Creative Commons Attribution Non-Commercial License (https://creativecommons.org/licenses/by-nc/4.0) which permits unrestricted non-commercial use, distribution, and reproduction in any medium, provided the original work is properly cited. behavioral therapy [2].

ASD was first reported by Kanner in 1943 and recognized as a distinct disorder in the 1970-80s. This group of disorders is mostly treated with special education and behavioral therapy, which can improve social interaction, including communication.

The importance of early intervention in ASD has been emphasized [3], but the definitions and composition of early intervention are still unclear. Specifically, the timing of intervention is uncertain, whether 'early' is relative to the time of diagnosis, or whether intervention needs to be started before 3 years of age. According to a report on the state of focused early interventions in South Korea, special education and behavioral therapy are provided for over 20 hours/week for children aged 2-3 years [4]. Overseas, the National Research Council recommends 15-25 hours/week of therapeutic intervention for children aged between 18 months and 5 years [5]. The uncertainty regarding the definitions and methods of early intervention reflects the lack of research on specific treatment methods and their effectiveness, which, in turn, is because these studies are difficult to undertake [3]. In particular, there are obstacles in evaluating early interventions effectiveness, that can be readily measured. And there are issues of generalization because the treatment effects sometimes are not sustained in daily living outside of the structured treatment environment [6]. When the effects of applied behavior 
intervention were analyzed based on data from previous studies, the effects were unclear, and the authors concluded that further research was needed [7].

In spite of these restrictions, several studies have reported that early diagnosis and intervention for ASD results in positive effects in the later course of the disorder $[8,9]$. When testing the effectiveness of a treatment, randomized controlled trials (RCTs) provide stronger evidence than single-group studies or case reports. Therefore, in this study, we reviewed RCTs related to early intervention for ASD.

\section{METHODS}

The current study was limited to interventions based on behavioral therapy and social skills training as early interventions for ASD in RCTs. Studies were restricted to original articles with a publication date between 2000 and 2017. This range was selected based on the considerations that RCTs after 2006 were reported when reviewing the American Academy of Child and Adolescent Psychiatry (AACAP) practice parameters in 2014 [3], and that the previous AACAP ASD guidelines [10] were published in 1999.

\section{Literature search and selection}

We used the following procedure to select studies for analysis in this review. For search terms, we defined the disorder name as 'autism,' research methods as 'randomized/randomized,' 'controlled,' and 'RCT' and the subjects and intervention methods as 'early,' 'child,' 'children,' and 'intervention.' Then we searched using combinations of these terms. For the search engine, we used Pubmed. We restricted the search to original articles written in English. We excluded systematic reviews, meta-analyses, and review articles. The initial literature search returned 810 studies. Of these, we excluded duplicates, and we selected 41 studies that met the following conditions based upon the inspection of the titles and abstracts. The inclusion criteria were: 1) participants aged 6 years or younger and diagnosed with autism or ASD; 2) RCT design; and 3) comparison groups include at least 10 persons each. The exclusion criteria were: 1) studies of participants with cerebral palsy, epilepsy, or genetic disease; 2) studies on comorbid psychiatric disorder or accompanying symptoms; 3) studies of the high ASD risk groups; 4) studies testing the efficacy of substances, drugs, or alternative therapy; 5) studies testing the efficacy of art therapy, music therapy, transcranial magnetic stimulation, therapy using robots or multimedia, biofeedback therapy, or auditory therapy; and 6) case reports, open trials, or reports of long-term follow-up observations. After inspecting the full text of the 41 studies, we finally selected 18 original articles that met the inclusion/exclusion criteria.

\section{RESULTS}

We classified the selected studies based on the identity of the person providing treatment directly to the children with ASD. There were 12 studies where the intervention was administered by the parents or guardians, 4 studies where treatment was provided directly by a therapist, and 2 studies where the intervention was administered by a school teacher or teaching assistant. Among interventions in which treatment was provided directly by a therapist, studies with and without training for parents were both included.

The early interventions reported in the 18 studies were broadly based on behavioral therapy but depending on the techniques or principles presented for the main treatment methods, these were divided into 'applied behavioral analysis (ABA) early intervention,' 'joint attention (JA) early intervention,' and 'social skill-communication (SC) early intervention.' There are overlaps between these classifications, since JA can be included as social skills, and ABA can improve social skills; nevertheless, for the purposes of this review, we arbitrarily divided early interventions according to the principles and technique emphasized by the authors. This classification is largely consistent with previous research, in which interventions were divided into behavioral, social-communication focused, and multimodal developmental interventions [11]. There were 3 studies using ABA early intervention, 8 studies using JA early intervention, and 7 studies using SC early intervention. Among the 18 studies, several studies tested the effectiveness of the same early interventions under different circumstances; in these cases, rather than selecting one representative study, we included all the studies in our analysis.

\section{ABA early intervention (Table 1)}

Dawson et al. [12] administered the Early Start Denver Model (ESDM), an early intervention based on the principles of ABA, to toddlers with ASD aged 18-30 months. Compared to the control group, who only received a general intervention that could be administered in the local community, the ESDM group showed significant improvements in intelligence, language, adaptive behavior, and changes in autism diagnosis. The treatment schedule was 2 hours/session, 2 sessions/day, 5 days/week and was provided directly by a therapist. The parents received education regarding the treatment twice per month and participated in the treatment, as well. The ESDM group showed a mean increase of 17.6 in intelligence scores, where intelligence and language were measured using the Mullen Scales of Early Learning. One study examined the effects of early intervention on language development based on pivotal response treatment (PRT), and reported improvements in language development and adaptive communica- 


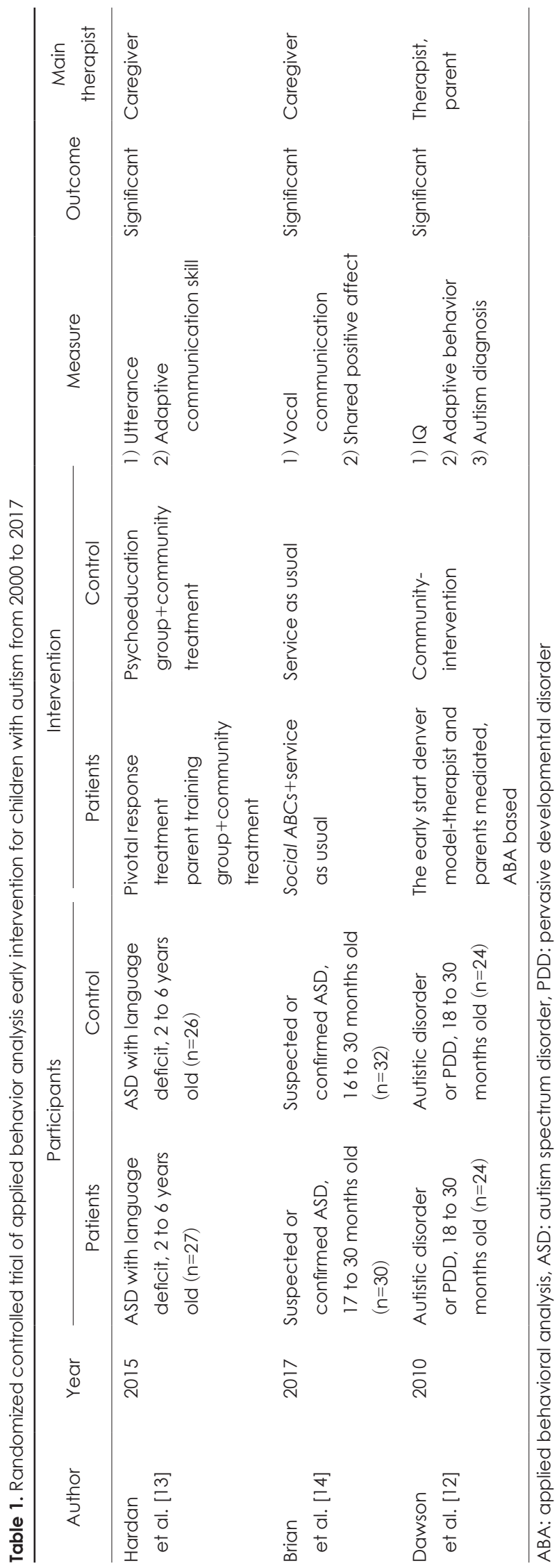

tion skills. The participants in this study [13] were autistic children with language deficits between 2 and 6 years old. The intervention was performed through a usual local treatment service, and the direct intervention was administered every week for 12 weeks by parents who had received instruction from a therapist. The control group received education for parents of children with ASD for the same duration. The usual local serive also were provided to them.

There was also an early intervention for ASD that aimed to promote emotional reciprocity, functional communication, and positive affect sharing with others. This intervention, the Social ABCs, used the PRT technique and is based on $\mathrm{ABA}$. This intervention was administered via the parents to ASD toddlers aged 16-30 months. The intervention was added to a usual treatment service. Compared to the control group that only received usual treatment, the toddlers in intervention group showed significant improvements in vocal communication [14]. However, of the total 62 participants in this study, 42 children had a confirmed diagnosis of ASD, while the other 20 children only had significant ASD-related symptoms at the beginning of the study.

\section{JA early intervention (Table 2)}

A study conducted by Drew et al. [15] administered an intervention for 12 months to pre-school children with a mean age of 23 months through parental training focused on JA. The intervention was performed in addition to general local therapeutic interventions. Compared to the group that received general local services alone, the intervention group showed significant improvements in language according to a parent-reported assessment.

There was one follow-up study using an early intervention based on the intervention described by Drew et al. [15]. In this study [16], an intervention focusing on JA and language skills was administered through parental training. Compared to the control group, the intervention group showed no differences in language or overall improvements. Specifically, the intervention was administered to autistic toddlers aged 12-42 months at home for a duration of 12 months by caregivers who had received instruction from a therapist. The therapist's caregivers was provided for 2 hours/week for 4 weeks, then for 3 hours at a home visit once every 6 weeks. At the home visit, the therapist and parent defined target behaviors, and the parents received feedback regarding the methods applied during parent-child playtime, and discussed the possibility of generalization in daily living. In addition, the therapist taught the parent techniques to enable the application of behavioral therapy principles, the use of non-verbal communication tools, and behaviors to promote interaction. Unlike the study by Drew et al. [15], this study 


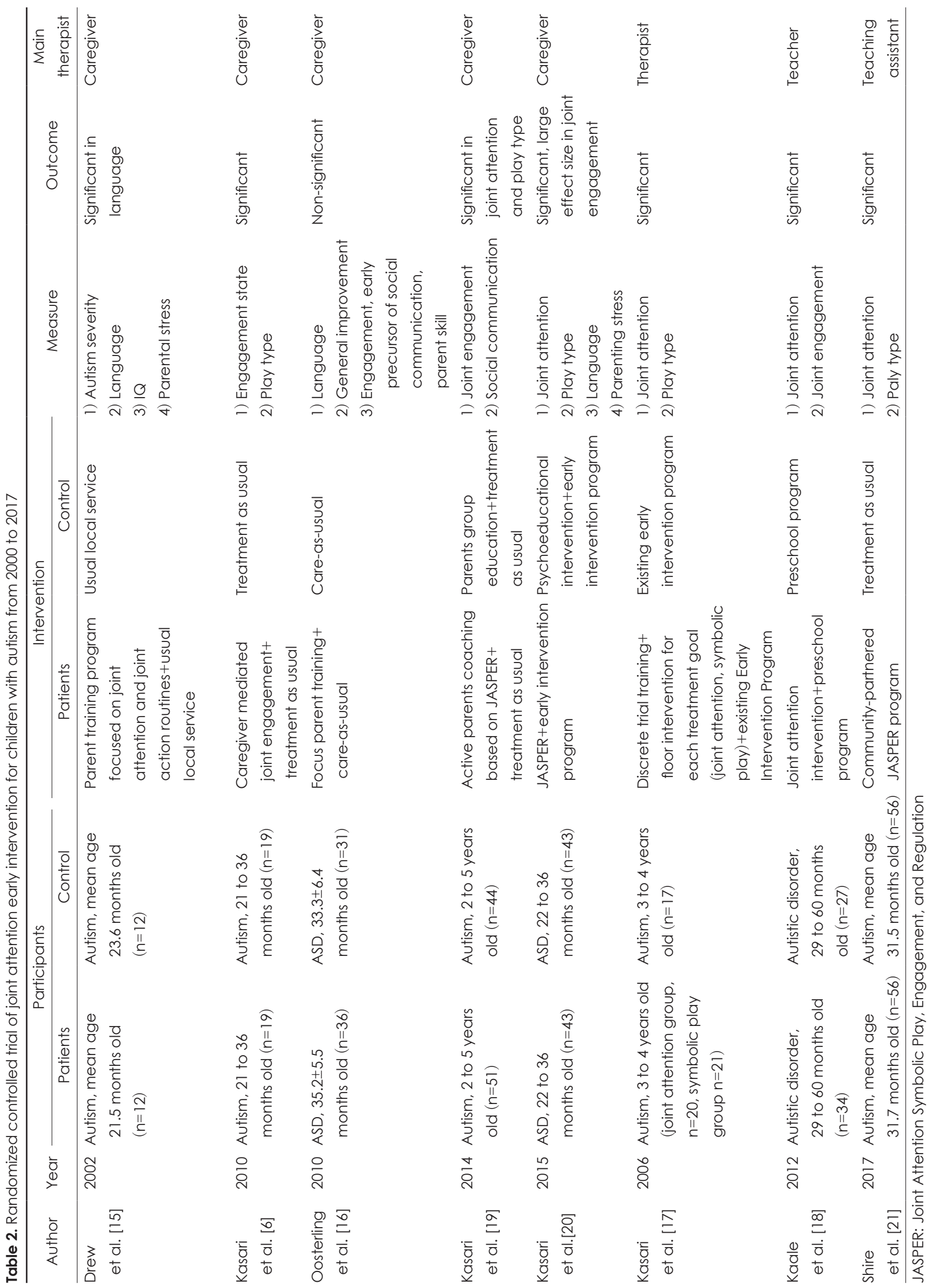


did not show any significant treatment effect. The authors proposed that this might be due to not only differences in the participants and study methods between two studies, but also the high standard of usual community service in their country, which was provided to both group in current study.

In 2006, Kasari et al. [17] performed an RCT on an early intervention using JA and play. Kasari et al. names later interventions based on these principles Joint Attention Symbolic Play, Engagement, and Regulation (JASPER), and 5 further studies were conducted after 2006 [6,18-21]. The study in 2006 produced an early intervention manual that aims to improve JA and symbolic play, based on the idea that autistic children showed delays or deficits in these areas, and implemented this manual for autistic children aged 3-4 years. The intervention was performed for 30 minutes/day, for 5-6 weeks. The JA intervention group contained 20 children, the symbolic play group contained 21 children, and the control group contained 17 children. Treatment goals were established for each group. In the actual intervention, the discrete trial training (DTT) technique was used for 5-8 minutes, based on $\mathrm{ABA}$, to train the target behaviors through prompting and reinforcement at a table. Thereafter, the target behaviors were trained in naturally occurring, semi-structured situations on the floor. The basic principles applied here were to follow the child's interests and lead, to talk about what the child was doing, or to repeat back what the child said. Other principles were to expand on the child's conversation and provide corrective feedback, to sit close to the child and make eye contact, and to create environmental adjustments to engage the child. These principles, based on previous studies, encouraged imitating the child's behaviors towards toys and used the child's interests to develop play. In the JA group, target behaviors were divided into behaviors initiating JA and responding to JA, and the target behaviors in these categories were then further subdivided. Similarly, in symbolic play, target behaviors were defined by the specific stage of play. These target behaviors were trained repeatedly in treatment. As a result, the respective groups showed significant changes in JA and play behaviors compared to the control group.

Kasari et al. [6] examined effects of a caregiver mediated joint engagement intervention for autistic toddlers aged 2135 months in 2010. In this study, the intervention consisted of 24 sessions, with 3 sessions/week for 8 weeks. In this intervention, a therapist instructed the caregivers in child-caregivers interactions, and the intervention was implemented by the caregivers. This treatment was based on the principles in the floor of intervention from the 2006 study by Kasari et al. [17]. Each session was 40 minutes, and the program was composed of 10 -session modules. The study assessed the effects of the intervention before and after treatment, and after 1 year. The change of engagement state and play type were two main outcomes. Engagement state was assessed using the categories of 'unengaged/other engagement,' 'object engagement,' and 'joint engaegemnt.' Play type was divided into functional play and symbolic play. Compared to the control group, who only received usual intervention services in general circumstances, the frequency of joint engagement was higher in the intervention group, who also showed increased frequency of JA responses and functional play.

In a study conducted in Norway [18], a JA-based intervention was administered to autistic preschool children twice per day, 5 days/week, for 8 weeks, which made a total of 80 sessions. This intervention was administered by school teachers under the weekly guidance of a trained therapist based on the manual developed by Kasari et al. in 2006 [17]. Both the intervention group and the control group were provided with a preschool program, which is a professional full-day early education and care program provided to preschool children aged 1-5 years in the Norwegian community. The JA intervention was additionally provided to the intervention group. Compared to the control group who only received the preschool program, the intervention group showed a higher frequency of JA in interactions with teachers.

In one study of a JASPER early intervention [19] administered via parents to autistic children aged $2-5$ years from low resourced families, the intervention was performed for 2 hours/week for 12 weeks. For the intervention group, a therapist instructed caregivers about daily living based on the principles of the JASPER intervention in order to increase JA and symbolic play. This process was conducted at home. For the control group, only the caregivers were educated for the same duration. After the intervention, the intervention group showed changes in JA and play patterns. This study did not measure intelligence, language, or adaptational function to assess the treatment effects.

Later, in 2015, in a study comparing the effects of a parentmediated JASPER intervention with parent psychoeducation [20], joint engagement showed an improved pattern in the JASPER intervention group. Both groups were provided usual early intervention for toddlers with autism.

In a study examining the effectiveness of JASPER intervention in the local community [21], the JASPER intervention group showed significant improvements in JA and play behaviors compared to a usual treatment group. In this study, teaching assistants were supervised by trained therapists, and the intervention was performed for 10 weeks for toddlers with a mean age of 31 months.

Social skill-communication early intervention (Table 3) A study by Aldred et al. [22] examined the effects of an in- 


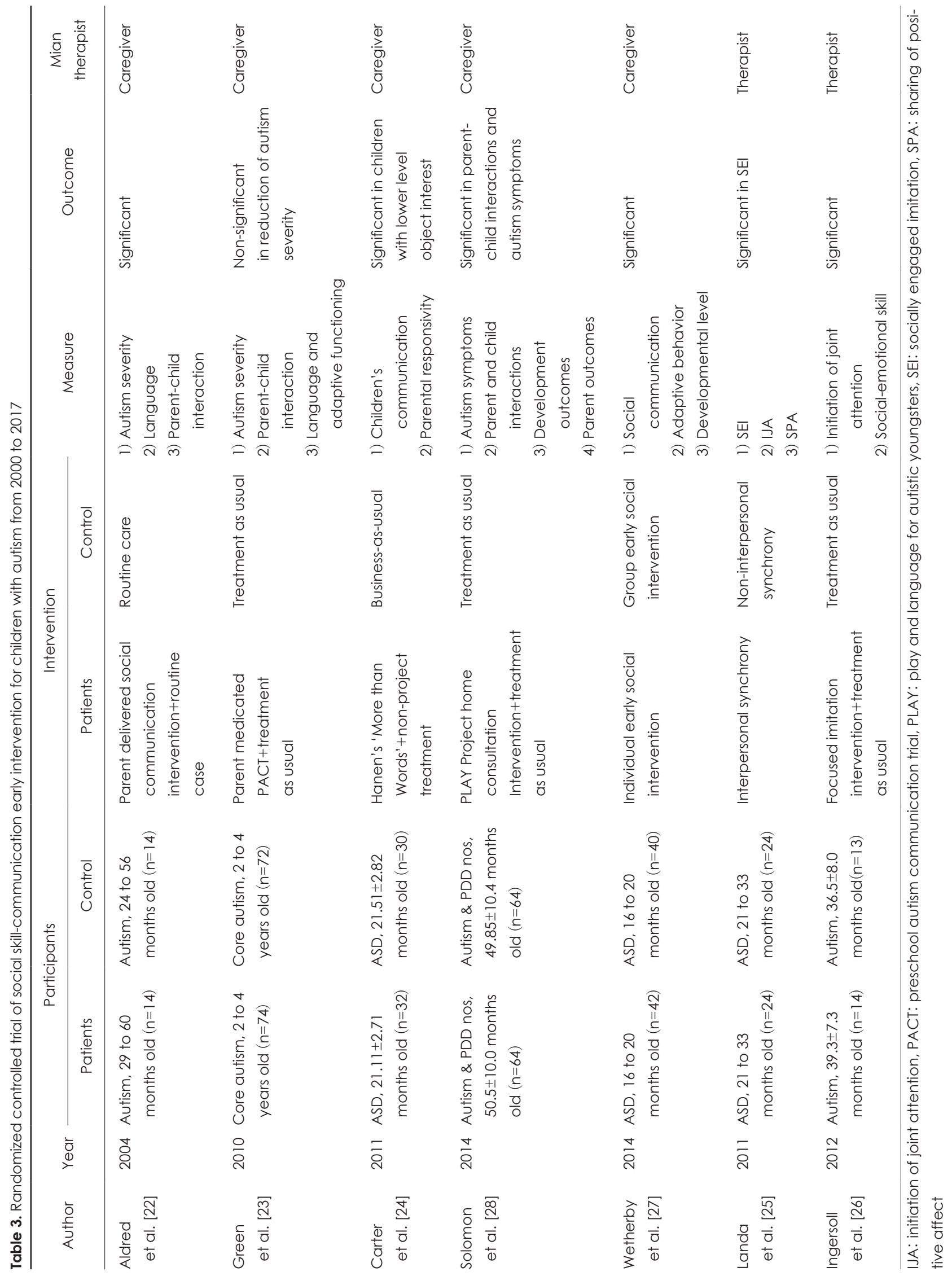


tervention to improve communication during play between parents and children. In this study, the usual intervention service was maintained, and a social communication intervention was added for children with autism aged 29-60 months. This intervention was performed directly by the parents. The parents and child attended monthly treatment sessions for 6 months, followed by a further 6 months of less frequent maintenance sessions. The intervention group received regular therapist contact once per month for 6 months, and thereafter, once every 2 months for another 6 months. When the two groups were compared after 12 months, the intervention group showed improvements in autistic symptoms assessed using the autism diagnostic observation schedule (ADOS), as well as improvements in expressive language and parent-child interactions.

Green et al. [23] examined the effects of the parent-mediated preschool autism communication trial (PACT) intervention, which focuses on communication. The participants in this study were 146 children between the ages of 2 years and 4 years 11 months, who were evaluated for severity of ASD according to the ADOS-generic (ADOS-G) and autism diagnostic interview-revised (ADI-R). This intervention consisted of methods to first improve the sensitivity and responsiveness of parents to the child, and then to increase communication with the child using strategies such as action routines, familiar repetitive language, and pauses. The intervention was implemented by the parents, who were guided through the 18sessions by a therapist. Each session was 2 hours long, and for the first 6 months, there was 1 session every 2 weeks. Thereafter, there was 1 session/month for another 6 months. The control group continued to receive the usual intervention provided in the community. When the changes in the two groups were compared 13 months after the beginning of treatment, there was no difference in autism symptom severity, but parent-child interactions showed improvements in the intervention group. The effects on language were small and the two groups showed no difference in language ability measured with a standardized instrument. However, the intervention group did show an improvement in parent-rated language. The authors of this study reported that the PACT intervention had no effect on reducing autism symptoms.

Hanen's 'More than Words' intervention is conceived to improve communication in ASD. This intervention is implemented directly by the parents under the guidance of a therapist. When this intervention was applied to ASD toddlers aged 15-25 months, there was an improvement in communication ability during daily routines compared to a control group who only received usual treatment [24]. In this intervention, the therapist provides education to the parents in 8 group sessions, and in between these 8 sessions, there are
3 individual parent-child sessions at home.

When a social communication intervention was added to supplement a preexisting comprehensive early intervention, compared to a group that simply continued the preexisting intervention, there were improvements in social communication. In this study [25], DTT, PRT, routine-based interaction, visual cues, and low-tech augmentative communication systems were used for toddlers with ASD aged 21-33 months to additionally reinforce activities related to interactions with others, such as imitation, JA, and positive affect sharing. Then, analysis was performed to verify whether social interactions improved. The additional activities consisted of imitating another person during social interactions, placing objects or pictures related to a situation on a wall, or imitating another person performing funny behaviors. The intervention was performed for approximately 2.5 hours/day, 4 days/ week for 6 months. The intervention was provided by a therapist in a classroom, and parent education was also provided to facilitate the continuation of these activities at home. The results showed an improvement compared to the control group in imitation in accordance with social situations.

Imitation is important in social development. One study developed Reciprocal Imitation Training and examined its effects on JA and social skills [26]; compared to a control group that only received usual treatment, the intervention group showed significant improvements. This intervention was administered by a therapist to ASD children between 27-47 months of age for 3 hours/week for a duration of 10 weeks. Although the intervention produced improvements in social function, the author of this study reported that the children's imitation was not a major mechanism of improvements through a mediation analysis and further studies will be required to understand the treatment components underlying the improvements.

Wetherby et al. [27] compared the effects of early social intervention provided by the parents either individually or in a group. The participants were toddlers diagnosed with ASD between 16-20 months old. This study educated the parents using manualized social communication, emotional regulation, and transactional support curriculum. Compared to the group therapy group, the individual therapy group showed significant effects on social communication, adaptive behaviors, and developmental level.

The play and language for autistic youngsters (PLAY) intervention is a parent-implemented intervention focusing on social interactions. This intervention is based on the developmental, individual differences, and relationship-based theory of Greenspan and Wieder. When the PLAY intervention was added to usual treatment for ASD children aged 2-5 years, there were significant improvements in parent- 
child communication and autism symptoms [28]. In this study, the caregivers received guidance from a PLAY consultant using coaching, modeling, and video feedback during 3-hour home visits every month. The consultant taught the parent to recognize and continually respond to the child's signals and guided the child to participate in interactions effectively. During modeling, the consultant showed the parent techniques through direct play with the child. Intervention goals and methods were modified each time depending on the child's developmental stage, and the duration and frequency were also adjusted. Families were encouraged to allocate 15-20 minutes of playtime several times a day, to achieve a total of approximately 2 hours of playtime per day.

\section{DISCUSSION}

When we reviewed RCTs on early intervention for ASD from 2000 to 2017, there several more studies than expected, and some studies used blinding methods in order to examine its effectiveness. Almost all studies used a design where the control group and intervention group both continually received a preexisting intervention or usual local treatment service, and the intervention group additionally received the experimental program to test its effects. Treatment duration varied from as little as a few weeks up to 2 years. Of the 18 studies we reviewed, 17 studies showed improvement in partial or all variables of interest compared to the control group; in one study [16], none of the measured variables showed any significant differences between the groups. Of the 18 studies, the intervention was implemented by the parents under the guidance of a therapist in 12 studies, directly by a therapist in 4 studies, and by schoolteacher in 2 studies. This shows the importance of parent training in early intervention.

In this review, we arbitrarily divided interventions into $\mathrm{ABA}, \mathrm{JA}$, and $\mathrm{SC}$ interventions based on the techniques and objectives emphasized by the authors. Many programs were based on principles of behavioral therapy and implemented interventions using methods such as play. The target behaviors included JA, play patterns, improved interaction with parents, language development, and parent responsiveness. Variables used to examine the effects of the interventions included changes in target behaviors, autism symptom severity, intelligence, adaptive behaviors, overall developmental level, and even parental stress.

In most studies, the variables directly related to the target behaviors showed significant improvements, but improvements in overall autism symptoms, intelligence, or language were limited. This appears to be because, in many studies, changes in the behaviors targeted by the experimental inter- vention were used as the efficacy outcome. For example, interventions targeting JA assessed increases in JA, or studies aiming to increase interactions with parents assessed interactions with parents. Given that the treatment process usually occurs in stages, the target behaviors will be better accomplished in the intervention group. These, poor JA and deficit in parent-child interaction, are characteristics that can be observed in ASD and are major targets of treatment, but if these changes cannot be generalized, or if the treated children do not show changes in overall development or autism symptoms, there will be limitations in assessing the effects of treatment. On the contrary, in a large study of ASD children with over 70 participants per group [23], the intervention was reported to show no effect in reduction of autism symptoms. Therefore, it may be necessary to measure overall or specific autism symptoms rather than only target behaviors, and to measure long-term changes rather than only shortterm changes immediately after treatment.

The study by conducted Carter et al. [24] showed, different effects depending on the characteristics of the ASD children, and there was an analysis seeking to understand the mechanisms for improvements [26]. This indicates that, through similar research designs or analyses, it will be possible to individualize early intervention treatments in the future.

In addition, early intervention studies use diverse terminology to express the similar meanings, and this can lead to difficulties in understanding and implementing the interventions in detail. In particular, given that 12 out of the 18 interventions were implemented directly by parents or caregivers, who are non-experts, this could be an even greater obstacle to the early intervention approach. Early intervention developers and researchers need to make efforts to clearly classify and define interventions using common, widely understood terms. In the case of interventions that do not belong to an existing classification, it will be necessary to introduce the principles and specific implementation methods in more detail.

\section{CONCLUSION}

There have been various RCTS between 2000 and 2017 regarding early interventions. There were a large number of interventions implemented by the parents under the guidance of an expert therapist. When we investigated behavioral and social skills therapy as early intervention for ASD, compared to the RCTs shown in existing guidelines [3], there were more studies pursuing improvements in JA, social interactions, social skills, and language development. In future studies, it will be necessary to measure the course of autism symptoms, 
language, intelligence, and overall development following interventions, as well as to investigate the long-term changes. Also, additional research will be needed to analyze differences in the effects according to the characteristics of the ASD children, and to investigate the mechanisms underlying improvements.

\section{Acknowledgments}

This study was supported by National Center for Mental Health.

\section{Conflicts of Interest}

The author has no potential conflicts of interest to disclose.

\section{ORCID iD}

Young-Hui Yang https://orcid.org/0000-0002-7095-6871

\section{REFERENCES}

1) American Psychiatric Association, DSM-5 Task Force. Diagnostic and statistical manual of mental disorders: DSM-5 ${ }^{\mathrm{TM}}$. 5th ed. Arlington, VA: American Psychiatric Publishing;2013.

2) Volkmar FR, Dykens EM, Hodapp RM. Intellectual disability. In: Martin A, Bloch MH, Volkmar FR, editors. Lewis's child and adolescent psychiatry: a comprehensive textbook. 5th ed. China: Wolters Kluwer;2018. p.434-442.

3) Volkmar F, Siegel M, Woodbury-Smith M, King B, McCracken J, State M; American Academy of Child and Adolescent Psychiatry Committee on Quality Issues. Practice parameter for the assessment and treatment of children and adolescents with autism spectrum disorder. J Am Acad Child Adolesc Psychiatry 2014;53:237-257.

4) Lee JY, Moon DS, Shin SH, Yoo HJ, Byun HJ, Suh DS. A survey on the status of hospital-based early intensive intervention for autism spectrum disorder in South Korea. J Korean Acad Child Adolesc Psychiatry 2017;28:213-219.

5) National Research Council. Educating Children with Autism. Washington, DC:The National Academies Press;2001.

6) Kasari C, Gulsrud AC, Wong C, Kwon S, Locke J. Randomized controlled caregiver mediated joint engagement intervention for toddlers with autism. J Autism Dev Disord 2010;40:1045-1056.

7) Spreckley M, Boyd R. Efficacy of applied behavioral intervention in preschool children with autism for improving cognitive, language, and adaptive behavior: a systematic review and meta-analysis. J Pediatr 2009;154:338-344.

8) Howlin P, Magiati I, Charman T. Systematic review of early intensive behavioral interventions for children with autism. Am J Intellect Dev Disabil 2009;114:23-41.

9) Warren Z, McPheeters ML, Sathe N, Foss-Feig JH, Glasser A, Veenstra-Vanderweele J. A systematic review of early intensive intervention for autism spectrum disorders. Pediatrics 2011;127:e1303-e1311.

10) Volkmar F, Cook E Jr, Pomeroy J, Realmuto G, Tanguay P. Summary of the practice parameters for the assessment and treatment of children, adolescents, and adults with autism and other pervasive developmental disorders. American academy of child and adolescent psychiatry. J Am Acad Child Adolesc Psychiatry 1999;38:16111616.

11) Tachibana Y, Miyazaki C, Ota E, Mori R, Hwang Y, Kobayashi E, et al. A systematic review and meta-analysis of comprehensive interventions for pre-school children with autism spectrum disorder (ASD). PLoS One 2017;12:e186502.
12) Dawson G, Rogers S, Munson J, Smith M, Winter J, Greenson J, et al. Randomized, controlled trial of an intervention for toddlers with autism: the Early Start Denver Model. Pediatrics 2010;125:e17-e23.

13) Hardan AY, Gengoux GW, Berquist KL, Libove RA, Ardel CM, Phillips J, et al. A randomized controlled trial of pivotal response treatment group for parents of children with autism. J Child Psychol Psychiatry 2015;56:884-892.

14) Brian JA, Smith IM, Zwaigenbaum L, Bryson SE. Cross-site randomized control trial of the Social ABCs caregiver-mediated intervention for toddlers with autism spectrum disorder. Autism Res 2017; 10:1700-1711.

15) Drew A, Baird G, Baron-Cohen S, Cox A, Slonims V, Wheelwright S, et al. A pilot randomised control trial of a parent training intervention for pre-school children with autism. Preliminary findings and methodological challenges. Eur Child Adolesc Psychiatry 2002; 11:266-272

16) Oosterling I, Visser J, Swinkels S, Rommelse N, Donders R, Woudenberg $\mathrm{T}$, et al. Randomized controlled trial of the focus parent training for toddlers with autism: 1-year outcome. J Autism Dev Disord 2010;40:1447-1458.

17) Kasari C, Freeman S, Paparella T. Joint attention and symbolic play in young children with autism: a randomized controlled intervention study. J Child Psychol Psychiatry 2006;47:611-620.

18) Kaale A, Smith L, Sponheim E. A randomized controlled trial of preschool-based joint attention intervention for children with autism. J Child Psychol Psychiatry 2012;53:97-105.

19) Kasari C, Lawton K, Shih W, Barker TV, Landa R, Lord C, et al. Caregiver-mediated intervention for low-resourced preschoolers with autism: an RCT. Pediatrics 2014;134:e72-e79.

20) Kasari C, Gulsrud A, Paparella T, Hellemann G, Berry K. Randomized comparative efficacy study of parent-mediated interventions for toddlers with autism. J Consult Clin Psychol 2015;83:554-563.

21) Shire SY, Chang YC, Shih W, Bracaglia S, Kodjoe M, Kasari C. Hybrid implementation model of community-partnered early intervention for toddlers with autism: a randomized trial. J Child Psychol Psychiatry 2017;58:612-622.

22) Aldred C, Green J, Adams C. A new social communication intervention for children with autism: pilot randomised controlled treatment study suggesting effectiveness. J Child Psychol Psychiatry 2004:45:1420-1430.

23) Green J, Charman T, McConachie H, Aldred C, Slonims V, Howlin P, et al. Parent-mediated communication-focused treatment in children with autism (PACT): a randomised controlled trial. Lancet 2010;375:2152-2160.

24) Carter AS, Messinger DS, Stone WL, Celimli S, Nahmias AS, Yoder P. A randomized controlled trial of Hanen's 'More Than Words' in toddlers with early autism symptoms. J Child Psychol Psychiatry 2011;52:741-752.

25) Landa RJ, Holman KC, O’Neill AH, Stuart EA. Intervention targeting development of socially synchronous engagement in toddlers with autism spectrum disorder: a randomized controlled trial. J Child Psychol Psychiatry 2011;52:13-21.

26) Ingersoll B. Brief report: effect of a focused imitation intervention on social functioning in children with autism. J Autism Dev Disord 2012;42:1768-1773.

27) Wetherby AM, Guthrie W, Woods J, Schatschneider C, Holland RD, Morgan L, et al. Parent-implemented social intervention for toddlers with autism: an RCT. Pediatrics 2014;134:1084-1093.

28) Solomon R, Van Egeren LA, Mahoney G, Quon Huber MS, Zimmerman P. PLAY Project home consultation intervention program for young children with autism spectrum disorders: a randomized controlled trial. J Dev Behav Pediatr 2014;35:475-485. 